\title{
Chaotic model for COVID-19 growth factor
}

\author{
Thiago de Salazar e Fernandes ${ }^{1}$
}

Received: 13 April 2020 / Accepted: 8 July 2020 / Published online: 26 July 2020

(C) Sociedade Brasileira de Engenharia Biomedica 2020

\begin{abstract}
Purpose The new COVID-19 pandemic caused by SARS-CoV-2 initiated in the world a large quarantine, due to the exponential capacity of the virus in spreading from human contact. In the present work, the dynamics of such spreading was evaluated by the analysis of the growth factor of the disease.

Method It was applied the space phase of the time series, the detrended fluctuation analysis (DFA) of the series, and the fractal dimension of the space phase.

Results It was possible to notice a strange attractor in the space phase of the growth factor indicating that the process is a deterministic chaos. The value of the alpha coefficient by DFA showed to be less than 0.5 , characteristic of anti-persistent long-range memory, in which large events alternate with small events, and vice-versa. The fractal dimension of the phase space set was a fractal number, between 1 and 2, another indicator that the growth factor of the disease is not random.

Conclusion This global analysis is pointing that the spread of COVID-19 is a deterministic chaos, with long-range memory. Understanding such dynamics may help the control of this disease.
\end{abstract}

Keywords Fractal analysis $\cdot$ Deterministic chaos $\cdot$ Detrended fluctuation analysis $\cdot$ Coronavirus $\cdot$ COVID-19

\section{Introduction}

Some processes in nature can be described as being nonlinear, which means that just small changes in the starting point can lead to enormous consequences. This is the case of the new infections by SARS-CoV-2 and the emergence of COVID-19 in the world. Just small changes in the genome of the first virus, SARS-CoV epidemics in 2002, resulted in a more severe spread and human infection of SARS-CoV-2, leading to the pandemic of COVID-19.

Recently, many countries in the world started the suppression of the virus by quarantine, with social distance of healthy individuals and isolation of the elders or those with clinical symptoms such as fever. Schools, works, and shopping centers were closed. If the governments do not act in suppression, but only in mitigation, the number of deaths would reach thousands in some countries and millions in the world (Ferguson et al. 2020).

Thiago de Salazar e Fernandes

thiago.fernandes@ufpe.br

1 Departamento de Biofísica e Radiobiologia, Universidade Federal de Pernambuco, Avenida Moraes Rego, 1235, Cidade Universitária, Recife, PE 50670-901, Brazil
Understanding exponential growth is difficult for human minds that evolved in a world in which daily tasks need only the linearity of events. It is hard to figure out that when we reach 30 steps in an exponential manner, we do not reach something linear to that such as 30 "something," but we are already in the scale of millions. Therefore, it is necessary to act early in prevention of SARS in order to avoid the collapse of the health system.

But if the understanding of exponential growth is already difficult to imagine for the human mind, imagine the understanding of a deterministic chaos behavior of many biological systems and epidemics. Chaos here does not mean the complete disorder of the system as the media is commonly associating this word with, but means when a system is determined by iteration of logistic equations resulting in unpredictable results (Mandelbrot 1983; Liebovitch 1998; Stam 2005; Kunicki et al. 2009). This means that even being very similar to random processes, or with an erratic behavior, a deterministic chaos presents self-similarity well known as resulting from fractal processes, and this means that there is a longrange correlation also known as the memory of the system (Peng et al. 1994; Stanley et al. 1996; Havlin et al. 1999; Wang et al. 2008; Peng et al. 1995). Therefore, chaos is the result of complex systems, when components of the system are correlated and competing for survival (such as rabbits and 
wolves, or fruit flies in a flask, sharks, and shrimp, and also virus and humans).

A chaotic system is deterministic but not necessarily predictable. What determinism means is that a small number of equations and its variables describe how the values in the past can be used to compute the values in the future. However, the sensitivity to initial conditions makes it not predictable in the long run, so that it cannot accurately predict the values of the variables far into the future (Liebovitch 1998).

In this work, the growth-factor of COVID-19 was evaluated by non-linear techniques, in order to better understand the pattern of the spread of this disease.

\section{Methods}

The data of growth factor of COVID-19 cases in the world that is calculated by every day's new cases divided by new cases on the previous day (from January 24 to April 9) was obtained from the website: https://www.worldometers.info/ coronavirus/coronavirus-cases/\#cases-growth-factor.

A growth factor higher than 1 indicates an increase of cases. Between 0 and 1 , it is a sign of a decline, and constantly above 1 is a sign of exponential growth.

This series was then submitted to the detrended fluctuation analysis (DFA). The DFA was calculated as the algorithm of Peng et al. (1994, 1995), using the software GNU Octave (https://www.gnu.org/software/octave/). The series was integrated and divided into boxes of equal length $(n)$. In each box of length $n$, a least-square line was fitted to the data representing the trend in that box. The $y$ coordinate of the straight line segments was denoted by $y n(k)$. Computing the DFA was performed according to Eq. 1:

$F(n)=\sqrt{\frac{1}{N} \sum[y(k)-y n(k)]^{2}}$

This computation was repeated over all the time scales (box sizes) to provide a relationship between the average root-mean fluctuation function $F(n)$ and the box size $n F(n)$ would increase with box size $n$. A power-law relationship on a double $\log$ plot indicates the presence of scaling. The fluctuations can be characterized by a scaling exponent alpha $(\alpha)$, the slope of the line relating $\log F(n)$ to $\log n: F(n) \sim n \alpha$, in order to provide a more accurate estimate of $F(n)$. If $\alpha=0.5$, the series is the result of a random event; $\alpha<0.5$ is pointing out that the series has an anti-persistent behavior, which means that an increasing trend in the past may imply a decreasing trend in the future, and vice-versa; $\alpha>0.5$ is indicating persistent long-range correlations (memory). The other values were as follows: $\alpha=1$ corresponded to $1 / \mathrm{f}$ noise (very rough landscape); the $\alpha \geq 1$, correlations existed but ceased to be of a power-law form or a random walk-like fluctuation; and finally, $\alpha=1.5-$ Brown noise, the integration of white noise (very smooth landscape).

The 2-dimensional phase space set was constructed from points with coordinates $X=x(n)$ and $Y=x(n+1)$. The image of the phase space set was submitted to the fractal analysis using the box counting method of the plugin "fracLac" of the software Image JTM, available for free download (https:// imagej.nih.gov/ij/plugins/fraclac/fraclac.html) and using the fractal dimension estimator (FDE), a software tool to measure the fractal dimension (FD) of a 2D image, available (http://www.fractal-lab.org/Downloads/FDEstimator.html). This method consists in the superposition of boxes in the image, where the lateral of the boxes is continuously reduced in size. In each of this reduction, the number of boxes necessary to cover pixels of the image is counted.

After this, the fractal dimension (FD) is calculated by Eq. 2:

$\mathrm{DF} \underset{r \rightarrow 0}{=\lim }\left(\frac{\log N r}{\log \left(1 /{ }_{r}\right)}\right)$

where $\mathrm{Nr}$ is the number of boxes necessary to cover the image in each progressive reduction of the side of the box $(r)$. FD will be the slope of the regression line, generated by the logarithm of the quantity of boxes in function to the logarithm of the size of the boxes.

\section{Results}

In Fig. 1, the time series of growth factor from January to April and of the growth factor of COVID-19 is shown.

The detrended fluctuation analysis (DFA) of the time series resulted in an alpha coefficient $(\alpha)$ of 0.27957 and a dimension of 2.7204 .

The data was reorganized in order to produce a phase space set, in which the next point of growth factor was plotted to the point in previous time (Fig. 2), showing a strange attractor characteristic of a deterministic chaotic process.

The fractal dimension of the attractor obtained in the phase space set was calculated by the fractal dimension estimator, and the value of FD was 1.33027 (Fig. 3).

When the fractal dimension was estimated by ImageJ software, the value of FD was 1.1623 .

\section{Discussion}

The growth factor of COVID-19 represents the number of new cases in relation to the previous day, from from January 24 to April 9. Growth factor above 1 is a sign of an increase of cases, between 0 and 1 is a decline, and constantly 1 is an exponential growth of cases. The results showed that the pattern is sometimes above 1 , but sometimes not. Are these 
Fig. 1 Growth factor of COVID19 from January 24 to April 9, 2020

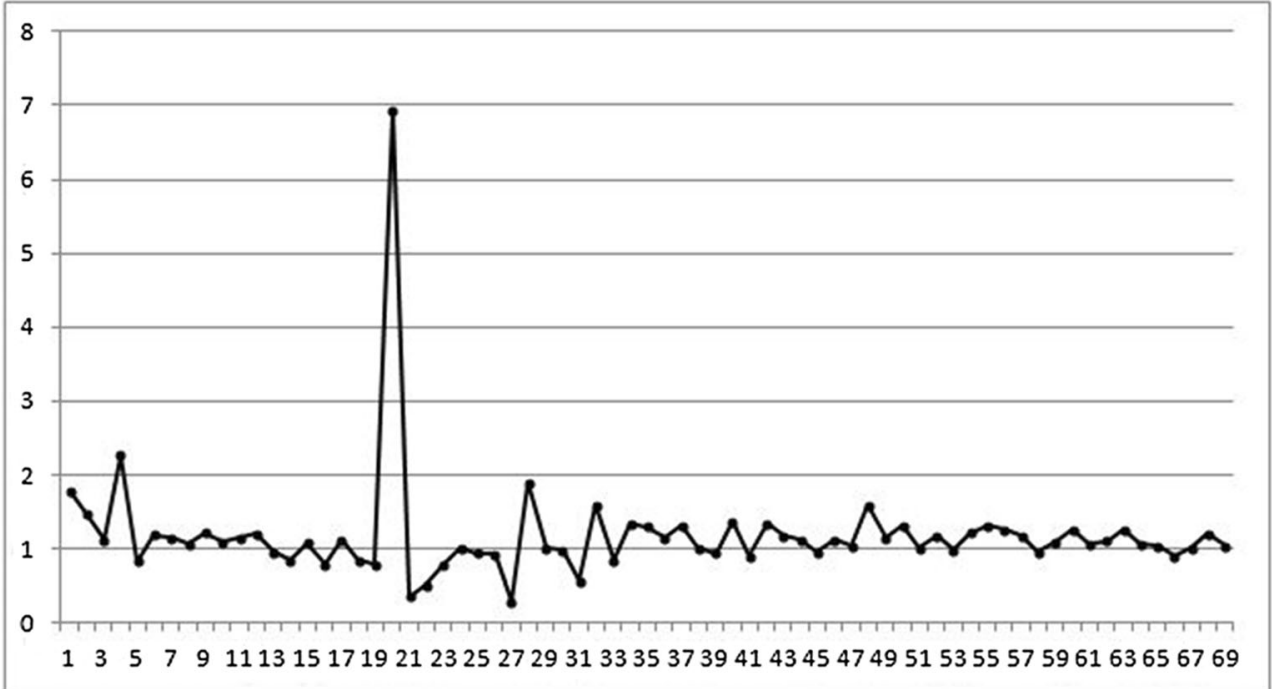

variations random, or are they due to the deterministic characteristics of how COVID-19 is transmitted from person to person?

The phase space set (Fig. 2) is a mathematical technique that allows identifying deterministic chaos and differentiating it from random processes. As it is possible to notice, there is a strange attractor characteristic of chaos, instead of a set of dots tending to fill all the space. The fractal dimension of the phase space set was a low number, closer to 1 , which means that the process is a deterministic chaos, or fractal. If the values of fractal dimension were closer to 2 , then the process would be characterized as random. Finally, the value of alpha DFA of the time series was lower than 0.5. It is well known that an alpha coefficient $(\alpha)$ from DFA between 0 and 0.5 corresponds to a power-law correlation such that large and small values of the time series are more likely to alternate (Peng et al. 1994, 1995), reinforcing the presence of self-similarity or long-range correlation (memory) in this time series (Mandelbrot 1983; Liebovitch 1998; Stam 2005; Kunicki et al. 2009).

Thus, the variation in the number of people with COVID19 showed to be due to a deterministic mechanism. The variables here will be associated with the rate of the transmission of the disease from one person to another, the duration of the incubation period before an infected person can transmit the disease and the duration of the infection period during which an infected person can transmit the disease (Liebovitch 1998).

The practical consequence of these results is that the small changes in the initial conditions can bring great changes in the dynamics of the spread of this disease. In other words, considering the spreading of this disease chaos as the data has
Fig. 2 Strange attractor of COVID-19 cases from January 24 to April 9

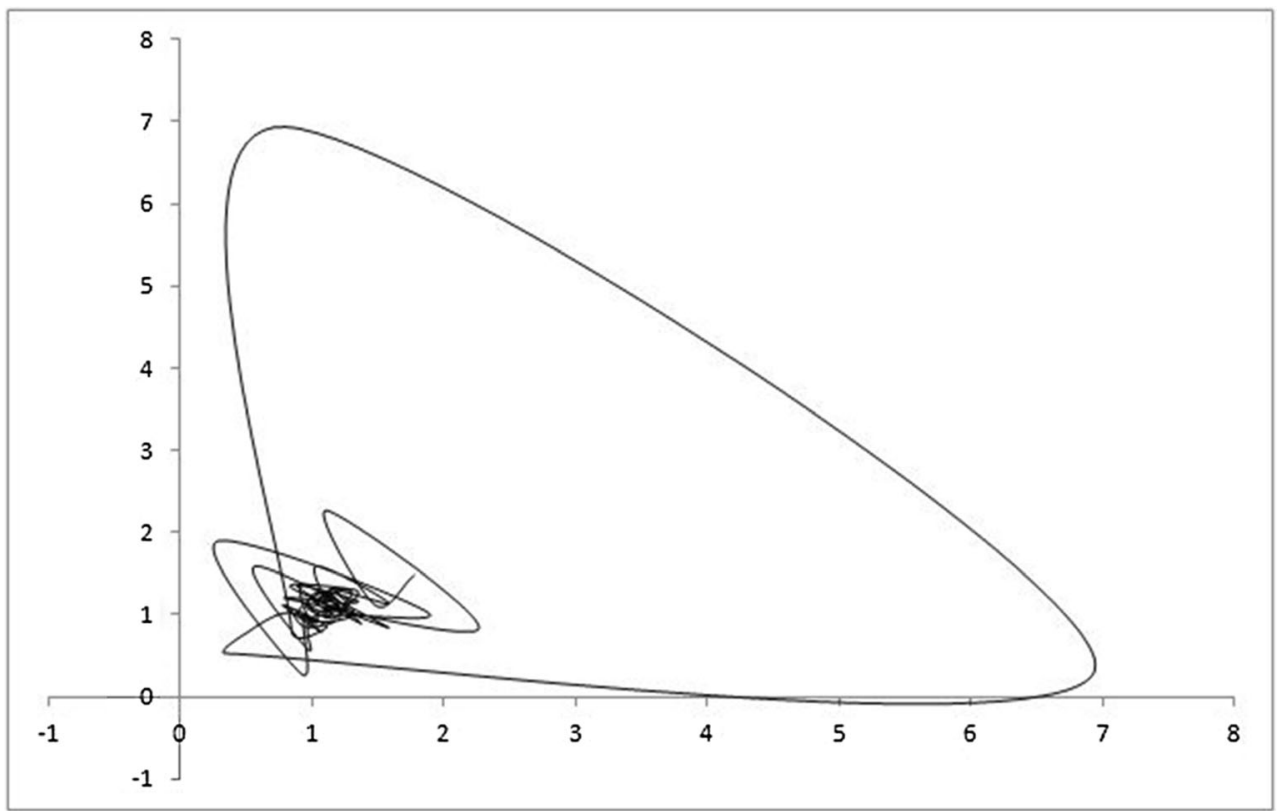


Fig. 3 Fractal dimension of the strange attractor of COVID-19 growth factor

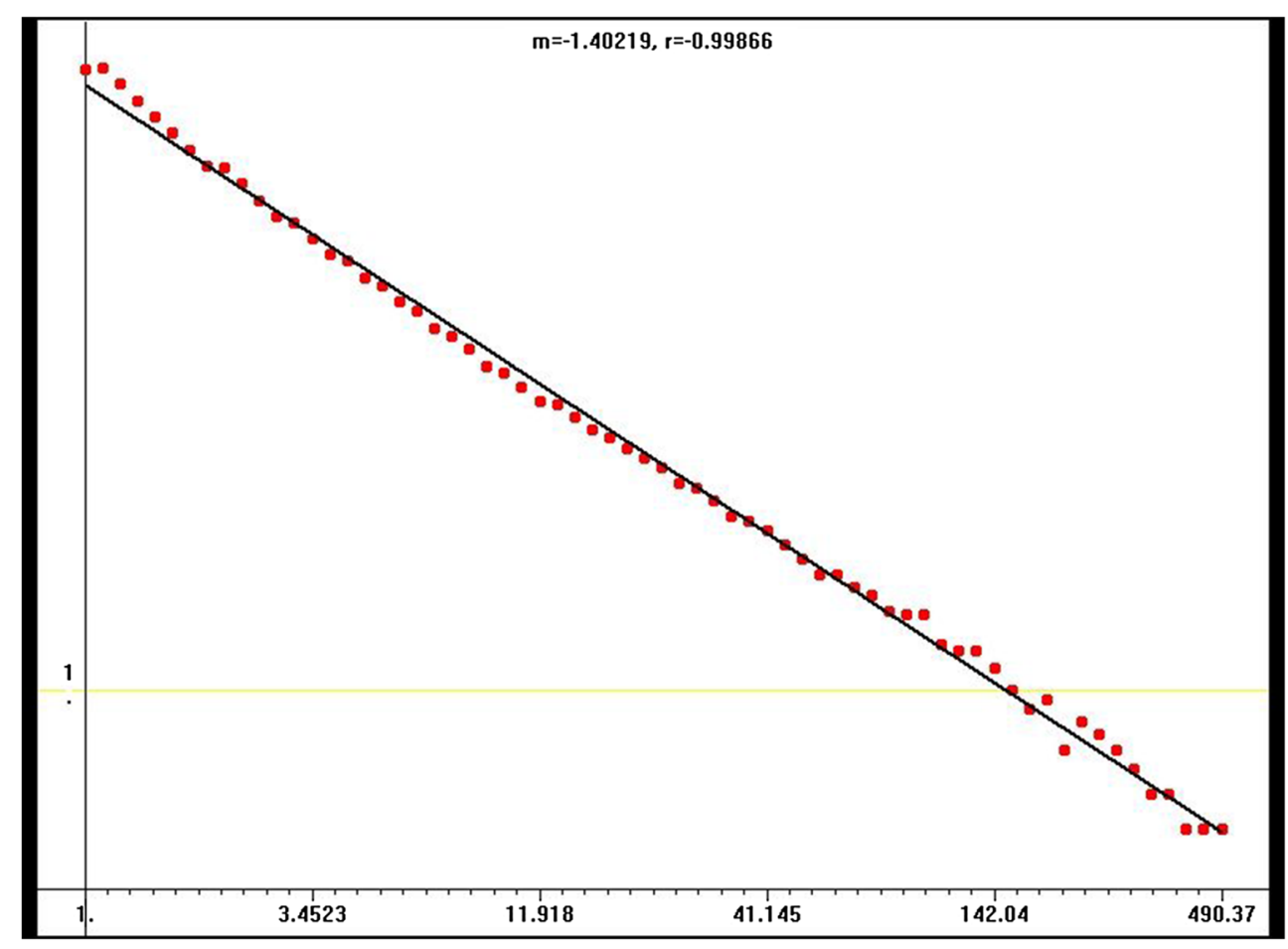

shown to be, changing initial conditions such as initiating social distancing may bring the spread of the disease to unexpected lower values. But also, in the opposite side of the same argument, if nothing is done, the epidemic can reach a catastrophic increase in the number of cases.

It is clear from the previous studies that the spread is exponential and suppression is the best way for controlling the high number of infected people that can lead to the collapse of health system (Ferguson et al. 2020). But considering the dynamics of the disease, the spread is much more complex, contamination is non-random from one person to another, and there is a deterministic chaos or fractal pattern behind the dissemination of this new virus, probably related to the human social contacts, contact among member of the family or the community, and so on. The dynamics of social contact is not like Brownian movement of molecules in a test tube, but a much more complex system because members of a community and society are more likely to be involved in a certain number of possible knots of relationships, such as colleagues of work, relatives, friends, social behaviors, and habits.

The peak in Fig. 1, also seen in the shift of the attractor in the phase space set in Fig. 2, probably corresponds to specific situations during the propagation of the pandemic. As the chaotic behavior is driven by the high sensitivity to initial conditions, without control, moderation during quarantine would promote to undetected people being in contact to others, leading to the exponential increase of the number of infections (Mangiarotti et al. 2016). Taking the chaotic behavior of epidemics into account is of utmost importance for disease control, especially for COVID-19 in which there are no vaccines or guaranteed treatment available yet in order to stop the pandemic.

\section{Conclusion}

The growth factor of COVID-19 shows a fractal pattern. This means that the growth of cases is oscillating from large values to small values, as a characteristic of a chaotic behavior, and not just by a constantly exponential growth. Understanding the complex dynamics of COVID-19 spread is crucial for decision-making, such as keeping social distance and finding new drugs and vaccines, and for a better prospective protection to this disease.

Acknowledgments The author would like to thank the Federal University of Pernambuco and Professor Romildo Nogueira, from the Federal Rural University of Pernambuco, for his discussions on chaos and fractals.

\section{Compliance with ethical standards}

Conflict of interest No conflict of interest.

\section{References}

Ferguson NM, Laydon D, Nedjat-Gilani G, Imai N, Ainslie K, Baguelin $\mathrm{M}$, et al. Impact of non-pharmaceutical interventions (NPIs) to reduce COVID-19 mortality and healthcare demand. Imperial college COVID-19 response: System; 2020. 
Havlin S, Buldyrev SV, Bunde A, Goldberger AL, Ivanov PC, Peng CK, et al. Scaling in nature: from DNA through heartbeats to weather. Phys A. 1999;273:46-9.

Kunicki ACB, Oliveira AJ, Mendonça MBM, Barbosa CTF, Nogueira RA. Can the fractal dimension be applied for the early diagnosis of non-proliferative diabetic retinopathy? Braz J Med Biol Res. 2009;42:930-4.

Liebovitch LS. Fractals and chaos simplified for life sciences. New York: Oxford University Press; 1998.

Mandelbrot BB. The fractal geometry of nature. 2nd. ed. New York: Ed. Freeman; 1983.

Mangiarotti S, Peyre M, Huc M. A chaotic model for the epidemic of Ebola virus disease in West Africa (2013-2016). Chaos. 2016;26: 113112.

Peng CK, Buldyrev SV, Havlin S, Simons M, Stanley HE, Goldberger AL. Mosaic organization of DNA nucleotides. Phys Rev E. 1994:49:1685-9.
Peng CK, Havlin S, Stanley HE, Goldberger AL. Quantification of scaling exponents and crossover phenomena in non-stationary heartbeat time series. Am Inst Phys. 1995;5:82-7.

Stam CJ. Nonlinear dynamical analysis of EEG and MEG: review of an emerging field. Clin Neurophysiol. 2005;116:2266-301.

Stanley HE, Afanasyev V, Amaral LAN, Buldyrev SV, Goldberger AL, Havlin S, et al. Anomalous fluctuations in the dynamics of complex systems: from DNA and physiology econophysics. Phys A. 1996;224:302-21

Wang SC, Li PC, Tseng HC. Long range correlation and possible electron conduction through DNA sequences. Phys A. 2008;387:5159-68.

Publisher's note Springer Nature remains neutral with regard to jurisdictional claims in published maps and institutional affiliations. 